\title{
Weighted DFT Codebook for Multiuser MIMO in Spatially Correlated Channels
}

\author{
Fang Yuan, Shengqian Han, Chenyang Yang \\ Beihang University, Beijing, China \\ Email: weiming383@gmail.com, sqhan@ee.buaa.edu.cn, \\ cyyang@buaa.edu.cn
}

\author{
Yu Zhang, Gang Wang, Ming Lei \\ NEC Laboratories, China \\ Email: \{zhang_yu,wang_gang, lei_ming $\} @$ nec.cn
}

\begin{abstract}
This paper proposes a novel codebook for multiuser multiple-input multiple-output systems under spatially correlated channels. Existing codebooks designed for correlated channels either require accurate channel statistics which is not favorable for practical systems, or only perform well in highly correlated channels. In this paper, we first analyze the per-user rate loss led by using DFT codebook, then propose a two-level codebook, named weighted DFT codebook. It consists of a DFT-based codebook and a Grassmannian linear packing codebook. The proposed scheme does not need accurate channel statistics and can adapt to both correlated and uncorrelated scenarios. Simulation results show significant performance gain of the proposed codebook over the existing codebooks in various correlated channels.

Index Terms-Limited feedback, MU-MIMO, WDFT, Codebook
\end{abstract}

\section{INTRODUCTION}

Limited feedback techniques in multiuser (MU) multipleinput multiple-output (MIMO) systems have received much attention in the last decade [1-4], where the design of codebook plays a key role.

When perfect channel state information (CSI) is known at the transmitter, MU-MIMO systems will have sum rate loss in spatially correlated channels [5]. Nonetheless, it is recognized recently that MU-MIMO systems with limited feedback perform even better in correlated channels than in uncorrelated channels [6]. This is because the spatial signature of the co-scheduled users is more separable in correlated channels hence the inter-user interference is able to be reduced.

For spatially correlated channels, it has been shown that Grassmannian linear packing (GLP) codebooks are not appropriate for MIMO systems any more [6]. Optimal codebook can be designed by using vector quantization techniques [7], but it requires high complexity. A systematic codebook was designed in [8], which achieves near perfect CSI performance even with few feedback bits when transmit correlation matrix is ill-conditioned and receive correlation matrix is wellconditioned. To reduce the complexity, a suboptimal codebook referred to as the transformed codebook is proposed, which can reuse the codebook designed for uncorrelated channels [3]. To achieve good performance, these limited feedback

This work was supported in part by NEC Laboratories, China, and by the International S\&T Cooperation Program of China (ISCP) (No. 2008DFA12100). schemes require accurate channel statistics information. DFT codebook does not need the feedback of channel statistics. It is shown that DFT codebook can achieve a near optimal sum rate in highly correlated channels $[9,10]$. However, DFT based codebooks do not perform well in less correlated channels.

In practice, it is desirable to design codebooks that apply for various correlated channels without the feedback of spatial correlation matrices. In this paper, we first analyze the per-user rate loss led by DFT codebook. The results indicate that DFT codebook is only efficient for quantizing channels with small angle spread (AS). For the channels with large angle spread, increasing the size of DFT codebook does not reduce the rate loss. This is owing to the fact that a single DFT codeword is not sufficient to represent a channel with large AS. Based on this observation, we propose a novel codebook for MUMIMO systems, named weighted DFT (WDFT) codebook. It is composed of two level codebooks, among which a DFTbased codebook is used to convey the statistical channel direction information (CDI), and a GLP codebook is applied to reflect the instantaneous fluctuation of fading channels. The proposed scheme can adapt to both correlated and uncorrelated scenarios. Simulation results demonstrate that the proposed codebook outperforms DFT codebook, GLP codebook, and a popular DFT-based two-level codebook for various correlated channels.

\section{SySTEM MODEL}

\section{A. MU-MIMO System with Limited Feedback}

We consider a downlink MU-MIMO system where a BS with $M$ antennas serves $K$ single-antenna users. The received signal at the $k$ th user can be expressed as

$$
y_{k}=\mathbf{h}_{k}^{H} \mathbf{x}+n_{k}, \quad k=1, \ldots, K,
$$

where $\mathbf{h}_{k} \in \mathbb{C}^{M \times 1}$ is the channel vector of the $k$ th user, $\mathbf{x}$ is the transmit signal vector of the $\mathrm{BS}$ to $K$ users, and $n_{k}$ is the additive white Gaussian noise with zero mean and unit variance.

Through capacity-limited uplink channel, each user feeds back quantized CDI and channel quality information (CQI) respectively for multiuser precoding and scheduling at the BS.

We propose to use a two-level codebook consisting of $\mathcal{C}_{1}$ and $\mathcal{C}_{2}$ for quantizing the statistical and instantaneous CDI, 
respectively. The codebook design and codeword selection issues will be discuss later.

\section{B. Correlated Channel Model}

We consider the widely applied virtual channel model [11] for spatially correlated channel. The channel vector of the $k$ th user is a superposition of $N$ uncorrelated subpaths, i.e.,

$$
\mathbf{h}_{k}=\sum_{n=1}^{N} a_{k, n} \mathbf{u}\left(\beta_{k, n}\right)
$$

where $a_{k, n}$ is the associated subpaths gain and $\mathbf{u}\left(\beta_{k, n}\right)=$ $\left[1, e^{-j 2 \pi \beta_{k, n}}, \ldots, e^{-j 2 \pi(M-1) \beta_{k, n}}\right]^{T}$ is the steering vector by assuming uniform linear array with omnidirectional antennas. Here, $\beta_{k, n}=\bmod \left(d / \lambda \sin \theta_{k, n}, 1\right) \in[0,1]$ is related to the physical angle of incidence $\theta_{k, n}$ with respect to the BS broadside, $d$ is the antenna spacing distance, $\lambda$ is the wavelength, and $\bmod$ is a mod operation. According to [11], the subpath gain $a_{k, n}$ is independent to the angle $\beta_{k, n}$.

The channel model can be rewritten more compactly as

$$
\mathbf{h}_{k}=\mathbf{U}_{k} \mathbf{a}_{k},
$$

where the direction matrix $\mathbf{U}_{k}=\left[\mathbf{u}\left(\beta_{k, 1}\right), \ldots, \mathbf{u}\left(\beta_{k, N}\right)\right]$, the gain vector $\mathbf{a}_{k}=\left[a_{k, 1}, \ldots, a_{k, N}\right]^{T}$ and channel average energy is normalized as $E\left\{\left\|\mathbf{h}_{k}\right\|^{2}\right\}=E\left\{M \sum_{n=1}^{N}\left|a_{k, n}\right|^{2}\right\}=1$ (\| $\|$ and || are respectively a norm and absolute operation).

\section{Per-user Rate Loss of DFT Codebook}

In this section, we analyze the performance of DFT codebook by deriving its per-user rate loss. The results will quantitatively show when DFT codebook is efficient for quantizing channels for MU-MIMO systems, i.e., when the performance of MU-MIMO systems can be improved by increasing codebook size. This fact has been ignored by most existing works.

We consider a DFT codebook uniformly sampling the angle domain as in [10]. The $i$ th codeword of such a $B$-bit DFT codebook $\mathcal{F}$ has the following structure

$$
\mathbf{f}\left(\phi_{i}\right)=\frac{1}{\sqrt{M}}\left[1, e^{-j 2 \pi \phi_{i}}, \ldots, e^{-j 2 \pi(M-1) \phi_{i}}\right]^{T},
$$

where $\phi_{i}=\frac{i}{2^{B}}\left(i=1, \ldots, 2^{B}\right)$ and $2 \pi \phi_{i}$ is the $i$ th sample in the angle domain $[0,2 \pi]$. Noting that DFT codebook is a single-level codebook, we can select a codeword $\boldsymbol{w}_{2}$ from $\mathcal{C}_{2}$.

Among all the multiuser beamforming schemes, zeroforcing beamforming (ZFBF) is considered here. Though suboptimal when user number is limited, it is of low complexity and is widely applied $[4,6]$. Based on the well-established upper bound of per-user rate loss for the case $K=M$ [4], the gap between the rate with perfect CSI and the rate with limited feedback when using ZFBF is

$$
\begin{aligned}
\Delta R_{\mathrm{up}}(P) & \leq E\left\{\log _{2}\left(1+\frac{P}{M} \sum_{k=1, k \neq i}^{M}\left|\mathbf{h}_{i}^{H} \mathbf{v}_{k}\right|^{2}\right)\right\} \\
& \leq \log _{2}\left(1+\frac{P}{M} E\left\{\left|\mathbf{h}_{k}\right|^{2}-\left|\mathbf{h}_{k}^{H} \mathbf{v}_{k}\right|^{2}\right\}\right) \\
& =\log _{2}\left(1+\frac{P(M-1)}{M} \mathcal{D}\left(\mathbf{h}_{k}, \mathcal{C}_{2}\right)\right),
\end{aligned}
$$

where $P$ is the transmit power at the BS, $\left|\mathbf{h}_{i}^{H} \mathbf{v}_{k}\right|^{2}$ is the interference term caused by the nonorthogonality of beamforming vector $\mathbf{v}_{k}$ to $\mathbf{h}_{i}$, and $\mathcal{D}\left(\mathbf{h}_{k}, \mathcal{C}_{2}\right)$ is the average distortion led by codebook $\mathcal{C}_{2}$ [3].

When DFT codebook is applied, i.e., $\mathcal{C}_{2}=\mathcal{F}$, the average distortion can be further upper bounded by (see appendix)

$$
\mathcal{D}\left(\mathbf{h}_{k}, \mathcal{F}\right) \leq M E\left\{c_{1} \min _{\phi_{i}} \sum_{n}^{N}\left|a_{k, n}\right|^{2}\left(\beta_{k, n}-\phi_{i}\right)^{2}+c_{0}\right\},
$$

where $\phi_{i}$ is the angle sample of codeword in $\mathcal{F}$. In brief, we denote the angle sample of the selected DFT codeword as $\bar{\beta}_{k}=\arg \min _{\phi_{i}} \sum_{n}^{N}\left|a_{k, n}\right|^{2}\left(\beta_{k, n}-\phi_{i}\right)^{2}$.

Two observations can be obtained from (5) and (6). On one hand, for the channels with small AS and the resolution of DFT codebook $2^{-B}$ exceeds the AS, we find that $\bar{\beta}_{k}$ satisfies $\left|\bar{\beta}_{k}-\beta_{k, n}\right|<2^{-B}$. Then we have

$$
\Delta R_{\text {up }}(P) \leq \log _{2}\left(1+\frac{P(M-1)}{M}\left(c_{1} 2^{-2 B}+c_{0}\right)\right),
$$

where $E\left\{M \sum_{n}\left|a_{k, n}\right|^{2}\right\}=1$ is used. In these scenarios, the rate loss is mainly constrained by the spatial resolution of DFT codebook, i.e. $2^{-B}$. Therefore increasing the codebook size $2^{B}$ is an effective way to improve the performance of MU-MIMO systems.

On the other hand, for the channels with large AS or the cases when the size of DFT codebook is large enough such that $\min _{m, n, m \neq n}\left|\beta_{k, n}-\beta_{k, m}\right| \geq 2^{-B}$ holds for all the subpaths, we will show that the sum rate of MU-MIMO systems can not be improved with the increase of the size of DFT codebook. To see this, we consider the case when $B$ approaches to infinity, then the angle samples $\phi_{i}$ of DFT codebook approach continuous values. In this case, we can obtain that $\bar{\beta}_{k}=\frac{\sum_{n}\left|a_{k, n}\right|^{2} \beta_{k, n}}{\sum_{n}\left|a_{k, n}\right|^{2}}$. By defining

$$
\sigma_{k}^{2}=\frac{\sum_{n}\left|a_{k, n}\right|^{2}\left(\beta_{k, n}-\bar{\beta}_{k}\right)^{2}}{\sum_{n}\left|a_{k, n}\right|^{2}},
$$

the rate loss in (5) is more tightly upper bounded by

$$
\Delta R_{\mathrm{up}}(P) \leq \log _{2}\left(1+\frac{P(M-1)}{M}\left(c_{1} E\left\{\sigma_{k}^{2}\right\}+c_{0}\right)\right) .
$$

Note that the term $E\left\{\sigma_{k}^{2}\right\}$ in (9) is the expectation of AS in virtual channel, thus the rate loss of DFT codebook increases with AS. This indicates that DFT codebook leads to nonnegligible quantization error in less correlated channels, which cannot be compensated by increasing the codebook size.

\section{WDFT CoDEBook DESIGN}

\section{A. Principles}

As shown in the previous analysis, the drawbacks of DFT codebook lie in that a single DFT codeword is not sufficient to represent the channels with large AS. In this section we propose a so-called WDFT codebook to resolve this problem.

Inspired by the structure of virtual channel model shown in (3), where the channel is expressed as a combination of the direction matrix $\mathbf{U}_{k}$ and the gain vector $\mathbf{a}_{k}$, we propose to 


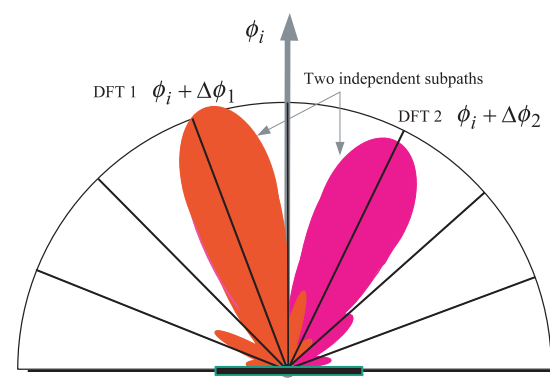

Fig. 1. Illustration of WDFT codebook design: using two DFT codewords to quantize a two-subpath channel.

use two-level codebook, $\mathcal{C}_{1}$ and $\mathcal{C}_{2}$, to respectively quantize the direction matrix $\mathbf{U}_{k}$ and the gain vector $\mathbf{a}_{k}$. Since a steering vector of uniform linear array has the same structure as a DFT codeword, we use multiple DFT codewords to construct the first level codebook $\mathcal{C}_{1}$, which targets to quantize multiple resolvable directions of the channel. According to the law of central limit, the gain of each resolvable direction approaches to a complex Gaussian vector when many subpaths are covered by each resolvable direction. Thus GLP codebook or Random vector quantization (RVQ) codebook can be used as the second level codebook $\mathcal{C}_{2}$. The principle of WDFT codebook is illustrated in Fig. 1, where the array response of a channel consisting of two subpaths is plotted, and two DFT codewords are used for quantizing the resolvable directions of two subpaths.

More specifically, the first level $B_{1}$-bit codebook $\mathcal{C}_{1}$ can be reconstructed by an original $B_{1}$-bit DFT codebook $\mathcal{F}$ with angle sample set $\mathcal{A}=\left\{\phi_{i} \mid \phi_{i}=\frac{i}{2^{B_{1}}}, i=1, \ldots, 2^{B_{1}}\right\}$. Codeword $\boldsymbol{w}_{1} \in \mathcal{C}_{1}$ constructed by $R$ DFT codewords $\mathbf{f} \in \mathcal{F}$ can be written in a matrix form as

$$
\boldsymbol{w}_{1}(i, \Delta \Phi, R)=\left[\mathbf{f}\left(\phi_{i}+\Delta \phi_{1}\right), \ldots, \mathbf{f}\left(\phi_{i}+\Delta \phi_{R}\right)\right],
$$

where $\Delta \phi_{r}(1 \leq r \leq R)$ are the relative deviations from the central direction $\phi_{i}$ of the codeword. Both the values of $\phi_{i}$ and $\Delta \phi_{r}$ are restricted within the discrete angle sample set $\mathcal{A}$, which is known by both the BS and users.

Define the deviation vector $\Delta \Phi=\left[\Delta \phi_{1}, \ldots, \Delta \phi_{R}\right]$, then the first level codebook depends on two parameters: $\Delta \Phi$ and $R$. The joint chosen of $R$ and $\Delta \Phi$ will be discussed in subsection IV-B.

Correspondingly, the $B_{2}$-bit second level codebook could be a GLP as in [1] or RVQ codebook as in [4] with proper dimension. The overall codeword in the proposed WDFT codebook can be generated by $\boldsymbol{w}_{1} \in \mathcal{C}_{1}$ and $\boldsymbol{w}_{2} \in \mathcal{C}_{2}$ as

$$
G\left(\boldsymbol{w}_{1}, \boldsymbol{w}_{2}\right)=\underbrace{\boldsymbol{w}_{1}}_{\text {size }} \underbrace{\boldsymbol{w}_{2}}_{M \times R \text { size } R \times 1} /\left\|\boldsymbol{w}_{1} \boldsymbol{w}_{2}\right\| .
$$

In practical scenarios, the resolvable directions vary slowly, while the gain coefficients on these directions change fast. To match the channel characteristic, in the WDFT scheme the first level codebook $\mathcal{C}_{1}$ can be used as a statistic CDI feedback while the second level codebook $\mathcal{C}_{2}$ can be used as an instantaneous feedback to reflect fast fading. Through this decomposition, the total feedback overhead is significantly reduced.

Since we employ GLP or RVQ as the second level codebook, we only need to design the first level codebook $\mathcal{C}_{1}$, which will be discussed in next subsection.

Given the codeword for statistical CDI $\boldsymbol{w}_{1}^{\text {opt }} \in \mathcal{C}_{1}$, the instantaneous codeword $\boldsymbol{w}_{2} \in \mathcal{C}_{2}$ can be selected at the user side as

$$
\boldsymbol{w}_{2}^{\text {opt }}=\underset{\boldsymbol{w}_{2} \in \mathcal{C}_{2}}{\arg \max }\left|\mathbf{h}_{k}^{H} G\left(\boldsymbol{w}_{1}^{\text {opt }}, \boldsymbol{w}_{2}\right)\right| .
$$

Denoting the channel vector space that is closest to the overall codeword $G\left(\boldsymbol{w}_{1}, \boldsymbol{w}_{2}\right)$ as

$$
\begin{aligned}
\mathcal{S}_{\boldsymbol{w}_{2}}^{\boldsymbol{w}_{1}}= & \left\{\mathbf{h}_{k}|| \mathbf{h}_{k}^{H} G\left(\boldsymbol{w}_{1}, \boldsymbol{w}_{2}\right)|\geq| \mathbf{h}_{k}^{H} G\left(\boldsymbol{w}_{1}^{\prime}, \boldsymbol{w}_{2}^{\prime}\right) \mid,\right. \\
& \left.\boldsymbol{w}_{1} \neq \boldsymbol{w}_{1}^{\prime}, \boldsymbol{w}_{2} \neq \boldsymbol{w}_{2}^{\prime}\right\},
\end{aligned}
$$

then the codeword for quantizing statistical CDI information can be selected as

$$
\boldsymbol{w}_{1}^{\mathrm{opt}}=\underset{\boldsymbol{w}_{1} \in \mathcal{C}_{1}}{\arg \max } \sum_{\boldsymbol{w}_{2} \in \mathcal{C}_{2}} \int_{\mathbf{h}_{k} \in \mathcal{S}_{\boldsymbol{w}_{2}}^{\boldsymbol{w}_{1}}}\left|\mathbf{h}_{k}^{H} G\left(\boldsymbol{w}_{1}, \boldsymbol{w}_{2}\right)\right|^{2} p\left(\mathbf{h}_{k}\right) d \mathbf{h}_{k},
$$

where $p\left(\mathbf{h}_{k}\right)$ is the probability density function (PDF) of channel vector.

\section{B. Optimal Design}

According to (10) and (11), constructing a WDFT codebook at the BS requires each user to feedback the parameters $\Delta \Phi$ and $R$. When the optimal values of $\Delta \Phi$ and $R$ are given, the optimal codebook $\mathcal{C}_{1}$ is then determined. To reflect the dependence of the codebook on these two parameters, we denote the overall codebook built by the first level codebook $\mathcal{C}_{1}$ and the second level codebook $\mathcal{C}_{2}$ as $\mathcal{G}(\Delta \Phi, R)$ in the following.

According to (5), the optimal codebook design problem minimizing the per-user rate loss upper bound can be formulated as

$$
\begin{array}{rl}
\min _{\Delta \Phi, R} & \mathcal{D}\left(\mathbf{h}_{k}, \mathcal{G}(\Delta \Phi, R)\right) \\
\text { s.t. } & R \in\left\{1,2, \ldots, 2^{B_{1}}\right\}, \\
& \Delta \Phi=\left[\Delta \phi_{1}, \ldots, \Delta \phi_{\mathrm{R}}\right], \text { with } \Delta \phi_{r} \in \mathcal{A}, r=1, \cdots, R .
\end{array}
$$

The design in (15), though optimal, is with a large search space which leads to a very high computation load as well as heavy feedback overhead. To address this issue, we consider a suboptimal candidate with equally spaced resolvable directions, i.e., $\Delta \phi_{r+1}-\Delta \phi_{r}=\Delta \phi$. Then the optimization problem in (15) reduces to

$$
\begin{array}{rl}
\min _{\Delta \phi, R} & \mathcal{D}\left(\mathbf{h}_{k}, \mathcal{G}(\Delta \phi, R)\right) \\
\text { s.t. } & R \in\left\{1,2, \ldots, 2^{B_{1}}\right\}, \\
& \Delta \phi \in\left\{2^{-B_{1}}, 2^{-B_{1}+1}, \ldots, 1 / 2\right\} .
\end{array}
$$

Compared with (15), (16) has much lower complexity since multiple parameters $\Delta \Phi$ in (15) becomes a single parameter 
$\Delta \phi$ in (16). This also leads to lower feedback overhead of channel statistics.

By contrast to the well-known Lloyd methods [7], the solution of the problem in (16) does not require explicit channel statistics at the BS. Only the feedback of $R$ and $\Delta \phi$ is enough for the construction of the overall WDFT codebook scheme.

\section{A Heuristic Method}

Unfortunately, although we can expect to deign codebooks with good performance by choosing $R$ and $\Delta \phi$ from the optimal problem in (16), it is feasible only when the PDF of channel vector is available at the user. Moreover, since $\mathcal{C}_{1}$ and $\mathcal{C}_{2}$ is coupled as in (12) and (14), it is hard to have a closed-form solution to the problem (16).

From practical application, a simpler scheme where $\mathcal{C}_{1}$ is directly designed according to the spatial correlation matrix $\mathbf{R}_{\mathbf{h}}=E\left\{\mathbf{h h}^{H}\right\}$ is highly preferred. Here, we propose a heuristic method for designing the first level codebook with only the information of spatial correlation matrix.

To this end, we view the problem shown in (16) as a statistical spatial fitting problem. Conceptually, the average CDI is less dependent on the gain vector. Therefore, we first separately find a CDI candidates set from the basic DFT codebook based on the spatial correlation matrix. Then, we strive for finding the optimal $R$ and $\Delta \phi$ based on the candidate set and given the constraint that the second level codebook is GLP or RVQ codebook.

In summary, the WDFT codebook feedback scheme can be implemented in the following two steps.

The first step is to find the first level codebook and codeword for each user given the second codebook:

1) Each user selects a DFT codeword as its average CDI from a $B_{1}$-bit DFT codebook $\mathcal{F}$ through

$$
i=\underset{j=1, \ldots, 2^{B_{1}}}{\arg \max }\left|\mathbf{f}\left(\phi_{j}\right)^{H} \mathbf{R}_{\mathbf{h}} \mathbf{f}\left(\phi_{j}\right)\right| .
$$

2) Then each user finds $R$ and $\Delta \phi$ as follows

$$
\begin{aligned}
\left\{\Delta \phi^{\mathrm{opt}},\right. & \left.R^{\mathrm{opt}}\right\}=\underset{\Delta \phi, R}{\arg \max }\left\{\max _{\boldsymbol{w}_{2} \in \mathcal{C}_{2}(R)} \mid \eta \boldsymbol{w}_{2}^{H} .\right. \\
& \left.\boldsymbol{w}_{1}^{H}(i, \Delta \phi, R) \mathbf{R}_{\mathbf{h}} \boldsymbol{w}_{1}(i, \Delta \phi, R) \boldsymbol{w}_{2} \mid\right\},
\end{aligned}
$$

where $\eta=\frac{1}{\left|\boldsymbol{w}_{1}(i, \Delta \phi, R) \boldsymbol{w}_{2}\right|}$ is a normalization factor, and the parameter $R$ and $\Delta \phi$ are constrained by (16b) and (16c).

The second step is feedback:

1) Each user feeds back the first level codeword $\boldsymbol{w}_{1}(i, \Delta \phi, R)$ in a long period, i.e., to feedback the index $i$ and the two scalar parameters $\Delta \phi$ and $R^{1}$.

2) Each user feeds back the second level codeword $\boldsymbol{w}_{2}$ to the BS in a short period, which is selected according to (11) and (12).

\footnotetext{
${ }^{1}$ In practice, the average CDI may change faster than the channel AS. In this scenario each user does not need to feedback $i, \Delta \phi$ and $R$ in the same period. By contrast, the user can feed back the later two parameters in a longer period, with which the BS obtains the first level codebook.
}

When the BS receives the two codewords, it can obtain the overall codeword by using (11). Note that such a solution does not require channel correlation matrix at the BS. Only the feedback of $R$ and $\Delta \phi$ is enough to construct the first level codebook.

\section{Simulation Results}

In the simulations, we evaluate the performance of the proposed WDFT codebook with Spatial Channel Model (SCM) [12]. The BS is equipped with a 4-antenna uniform linear array with a half wavelength antenna separation, which serves in total 10 single-antenna users by ZFBF based on limited feedback and greedy user scheduling [13]. The CQI is fed back as suggested in [14] and user speed is $3 \mathrm{~km} / \mathrm{h}$, which is commonly used for performance evaluation in MU-MIMO systems. Two typical scenarios are studied here: one is highly correlated scenario, which has an AS of 8 degrees as defined in [12], and the other is less correlated scenario with an AS of 35 degrees. In simulations, the first level codeword is fed back every $100 \mathrm{~ms}$ while the second level codeword is fed back every $5 \mathrm{~ms}$. In addition, a feedback delay of $1 \mathrm{~ms}$ is considered.

The first simulation is to compare the performance of the proposed codebook with existing codebooks under different feedback overhead. Besides the proposed WDFT codebook with optimal design in (16) and the heuristic solution, two schemes are also provided here for comparison: DFT codebook in [9], and a two-level DFT-based codebook proposed by SamSung in [15]. The basic idea of SamSung's codebook is using the first level statistic codebook to find a rough CDI range and using the second level instantaneous codebook to quantize the CDI within the range. Therefore, it is equivalent to a DFT codebook with more feedback bits.

For two-level codebooks including WDFT and SamSung's, the first level codebook has a size of $B_{1}=8$ bits. The size of the instantaneous codebook $B_{2}$ is the same for all codebooks, which is shown in the $\mathrm{x}$-axis. The average signal to noise ratio (SNR) is set to be $25 \mathrm{~dB}$. As shown in Fig. 2, for the channels with both 8- and 35-degree AS, the proposed WDFT codebook with both optimal design and heuristic solution outperforms the DFT codebook as well as the codebook proposed by SamSung. Moreover, with the increase of the second level feedback bits, a performance bottleneck for DFT and SamSung's codebook is observed in both scenarios, as indicated by our analysis in Section III.

The second simulation evaluates the performance of the proposed codebook and existing codebooks under different SNR for two different correlated channels. The codebooks in the simulation include the optimal WDFT codebook, the heuristic WDFT codebook, the SamSung's codebook and the GLP codebook proposed in [1]. DFT codebook is not shown here since it is inferior to the SamSung's scheme. The size of instantaneous codebook is set to be 6 bits. As shown in Fig. 3 , for the channels with both 8- and 35-degree angle spread, the proposed WDFT codebooks with both optimal design and heuristic solution outperform GLP and SamSung's codebooks. 


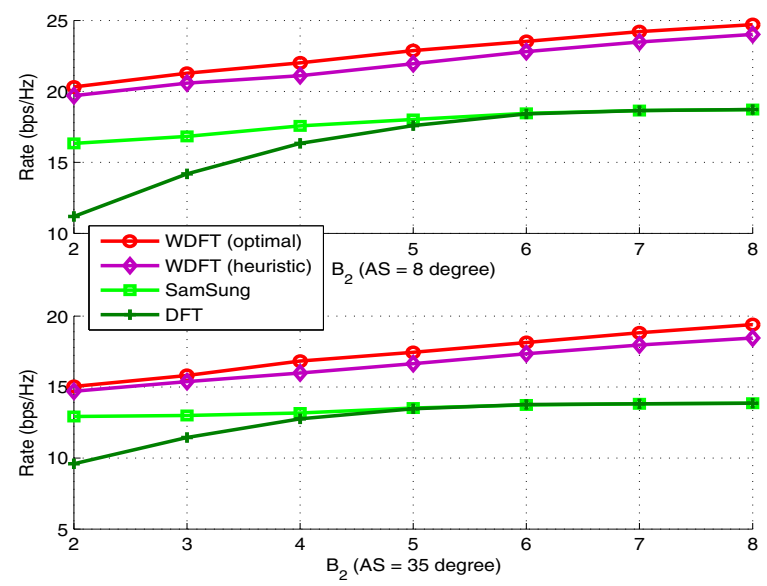

Fig. 2. Sum rate of different codebooks as a function of $B_{2}$ for two different correlated channels.

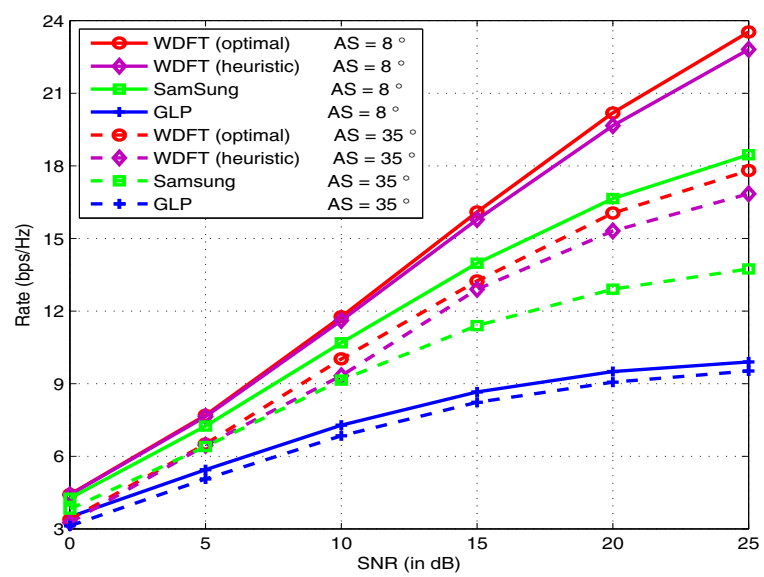

Fig. 3. Sum rate of different codebooks as a function of SNR for two different correlated channels $\left(B_{2}=6\right)$.

At the SNR of $25 \mathrm{~dB}$, a performance gain of $5 \mathrm{bps} / \mathrm{Hz}$ and 4 bps/Hz respectively achieved by optimal and heuristic WDFT can be observed over SamSung's codebook in both channels.

\section{CONCLUSION}

In this paper we have studied the codebook design method for correlated MU-MIMO channels. We propose a novel weighted DFT (WDFT) codebook, which can adapt to different correlated channel scenarios by judiciously designing feedback information for channel statistics. The overall WDFT codebook is simple and can be easily implemented for the prevalent MU-MIMO systems. Simulation results have shown that our method is effective in different channels without requiring too much feedback overhead.

\section{APPENDIX}

In the following, the average distortion led by codebook $\mathcal{F}$ is analyzed (the subscript $k$ is ignored for simplification).

$$
\begin{aligned}
\mathcal{D}(\mathbf{h}, \mathcal{F}) & \\
= & E\left\{|\mathbf{h}|^{2}\right\}-E\left\{\left|\mathbf{h}^{H} \mathbf{f}^{\mathrm{opt}}\right|^{2}\right\} \\
= & 1-\frac{1}{M} E\left\{\max _{\phi_{i}}\left|\sum_{n} a_{n}^{H} \mathbf{u}^{H}\left(\beta_{n}\right) \mathbf{f}\left(\phi_{i}\right)\right|^{2}\right\} \\
\leq & 1-\frac{1}{M} E\left\{\max _{\phi_{i}} \sum_{n}\left|a_{n}^{H} \mathbf{u}^{H}\left(\beta_{n}\right) \mathbf{f}\left(\phi_{i}\right)\right|^{2}\right\} \\
& +2 E\left\{\sum_{n} \sum_{m>n}\left|a_{n}^{H} a_{m}\right|\right\} \\
= & 1-\frac{1}{M} E\left\{\max _{\phi_{i}} \sum_{n}\left|a_{n}\right|^{2} \frac{\sin ^{2}\left(M \pi\left(\beta_{n}-\phi_{i}\right)\right)}{\sin ^{2}\left(\pi\left(\beta_{n}-\phi_{i}\right)\right)}\right\} \\
= & 1-c_{1} M E\left\{\max _{\phi_{i}} \sum_{n}\left|a_{n}\right|^{2}\left(\beta_{n}-\phi_{i}\right)^{2}\right\}+c_{0},
\end{aligned}
$$

where channel energy $E\left\{|\mathbf{h}|^{2}\right\}$ is normalized to one in (17a) and $c_{0}=2 E\left\{\sum_{n} \sum_{m>n}\left|a_{n}^{H} a_{m}\right|\right\}$. In the above derivations, (17b) is followed by the facts that $\left|\sum_{n} x_{n}\right|^{2} \geq \sum_{n}\left|x_{n}\right|^{2}-$ $2 \sum_{n} \sum_{m>n}\left|x_{n} x_{m}\right|$ and $\left|\mathbf{u}^{H}\left(\beta_{n}\right) \mathbf{f}\left(\phi_{i}\right)\right| \leq \sqrt{M}$. In (17c), the inequality $\frac{\sin ^{2}(M \pi x)}{\sin ^{2}(\pi x)} \geq M^{2}\left(1-c_{1} x^{2}\right)$ is applied $\left(c_{1} x^{2}<1\right)$, where $c_{1}=-\left.\frac{d^{2} \sin ^{2}(M \pi x)}{d x^{2} M^{2} \sin ^{2}(\pi x)}\right|_{x=0}=\frac{2\left(M^{2}-1\right)}{3} \pi^{2}$, which is not hard to be derived.

\section{REFERENCES}

[1] D. J. Love, J. Heath, R. W., and T. Strohmer, "Grassmannian beamforming for multiple-input multiple-output wireless systems," in Proc. IEEE Int. Communi. Conf. (IEEE ICC), vol. 4, 2003.

[2] D. Gesbert, M. Kountouris, R. W. Heath, C.-B. Chae, and T. Salzer "From single user to multiuser: Shifting the MIMO paradigm," IEEE Signal Processing Mag., vol. 24, no. 5, pp. 36-46, 2007.

[3] D. J. Love and R. W. Heath, "Limited feedback diversity techniques for correlated channels," IEEE Trans. Veh. Technol., vol. 55, no. 2, pp. 718-722, 2006.

[4] N. Jindal, "MIMO broadcast channels with finite-rate feedback," IEEE Trans. Inform. Theory, vol. 52, no. 11, pp. 5045-5060, 2006.

[5] T. Y. Al-Naffouri, M. Sharif, and B. Hassibi, "How much does transmit correlation affect the sum-rate of MIMO downlink channels?" in Proc. IEEE Int Information Theory Symp (IEEE ISIT), 2006.

[6] B. Clerckx, G. Kim, and S. Kim, "Correlated fading in broadcast MIMO channels: Curse or blessing?" in Proc. IEEE Global Telecommun. Conf. (IEEE GLOBECOM), 2008.

[7] A. Gersho and R. M. Gray, Vector Quantization and Signal Compression. Kluwer, 1992.

[8] R. W. Heath, T. Wu, and A. C. K. Soong, "Progressive refinement for high resolution limited feedback multiuser MIMO beamforming," in Proc. 42nd Asilomar Conf. Signals, Systems and Computers, 2008.

[9] N. Ravindran, N. Jindal, and H. C. Huang, "Beamforming with finite rate feedback for los MIMO downlink channels," in Proc. IEEE Global Telecommun. Conf. (IEEE GLOBECOM), 2007.

[10] D. Yang, L.-L. Yang, and L. Hanzo, "DFT-based beamforming weightvector codebook design for spatially correlated channels in the unitary precoding aided multiuser downlink," in Proc. IEEE Int. Communi. Conf. (IEEE ICC), 2010.

[11] A. M. Sayeed, "Deconstructing multi-antenna fading channels," IEEE Trans. Signal Processing, vol. 50, no. 10, pp. 2563-2579, 2002.

[12] "Spatial channel model for MIMO simulations," 3GPP TR 25.996 V6.1.0, 2003.

[13] G. Dimic and N. D. Sidiropoulos, "On downlink beamforming with greedy user selection: performance analysis and a simple new algorithm," IEEE Trans. Signal Processing, vol. 53, no. 10, pp. 3857-3868, 2005.

[14] T. Yoo, N. Jindal, and A. Goldsmith, "Multi-antenna downlink channels with limited feedback and user selection," IEEE J. Select. Areas Commun., vol. 25, no. 7, pp. 1478-1491, 2007.

[15] "Views on the feedback framework for Rel. 10," SamSung, 3GPP TSG RAN 1, meeting \#61, R1-103377, 2010. 